\title{
Producción científica española en literatura desde una perspectiva de género a través de Web of Science (1975-2017)
}

\author{
Julia Haba-0sca* \\ Julia Osca-Lluch**

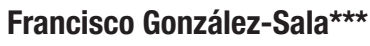

\begin{abstract}
Artículo recibido:
26 de julio de 2018

Artículo aceptado:

4 de diciembre de 2018

Artículo de investigación
\end{abstract}

\section{Resumen}

Se realiza un estudio comparativo desagregado por sexos de la producción científica en literatura de los investigadores que trabajan en instituciones españolas. Se han identificado 806 autores que han publicado algún trabajo de literatura en revistas indexadas en la WoS hasta el año 2017, analizando comparativamente su productividad y patrones de colaboración considerando la variable sexo. El $55.51 \%$ de los autores identificados fueron hombres frente a un $44.49 \%$ de mujeres. Los resultados muestran que entre los grandes productores del ámbito de la literatura predominan

* Facultad de Filología, Universitat de València, España

** Instituto de Gestión de la Innovación y del Conocimiento (CSIC-UPV), España

julia.haba@uv.es juosllu@ingenio.upv.es

*** Facultad de Psicología, Universitat de València, España 
los hombres. Entre los productores moderados (2-5 trabajos), el $50.36 \%$ de los autores identificados fueron hombres frente a un $49.64 \%$ de mujeres. Aunque predominan los trabajos realizados individualmente, como sucede en otras disciplinas científicas, se observa que hay un aumento de los trabajos realizados en colaboración a lo largo del tiempo, y aquellos en los que participan entre 3 o 4 autores están realizados por grupos formados por hombres y mujeres. Como conclusión se podría indicar que en la literatura española todavía no existe una igualdad de género, especialmente cuando se considera la élite de la investigación. Resulta fundamental profundizar y seguir realizando estudios que evalúen la evolución y tendencias de forma desagregada por sexos para acometer las medidas correctoras necesaria que permitan alcanzar la igualdad de género.

Palabras clave: Análisis de género; Literatura; Publicaciones Científicas; Análisis Bibliométrico

Spanish scientific production in literature through a gender perspective in the Web of Science (1975-2017) Julia Haba-Osca, Julia Osca-Lluch and Francisco GonzálezSala

\section{Abstract}

A comparative study disaggregated by sex about the scientific production in the literature of researchers working in Spanish institutions, has identified 806 authors who have published some literature work in journals indexed in the WoS until 2017, and analyzed comparatively their productivity and collaboration patterns considering the sex variable. It was found that $55.51 \%$ of the authors identified were men, compared to $44.49 \%$ of women. The results show that men predominate among the major producers in the field of literature. While among moderate producers (2 to 5) works, $50.36 \%$ of the authors identified were men, compared to $49.64 \%$ of women. Although works realized individually predominate, as it happens in other scientific disciplines, it is observed that there is an increase of works written in collaboration. I was also observed that collaborative works done between 3 or 4 authors are made up by groups formed by men and 
women. As conclusion it could be indicated that in the Spanish literature gender equality does not yet exist, especially when considering the elite of the research. It is fundamental to deepen and continue carrying out studies that evaluate the evolution and trends in a disaggregated way by sex, to undertake the corrective measures necessary to achieve gender equality.

Keywords: Gender Analysis; Literature; Scientific Publications; Bibliometric Analysis

\section{INTRODUCCIÓN}

— $\mathrm{n}$ la actualidad existe un creciente interés en conocer la situación de las mujeres en los diferentes ámbitos profesionales y académicos, por ello es necesario visibilizar numéricamente y conocer el estado de las mujeres en el ámbito científico (Huyer y Westholm, 2002). En los países de la Unión Europea, el porcentaje de mujeres graduadas en educación superior es superior al porcentaje de hombres graduados, excepto en Alemania, que tiene el valor más bajo de mujeres graduadas (47.9 \%). En España, en 2015, el porcentaje de mujeres graduadas en educación superior era $53.1 \%$ y el de hombres $46.9 \%$. Por campo de estudio, el mayor porcentaje de mujeres que se gradúan en España corresponde al campo de negocios, administración y derecho (11.5\%), mientras que los menores porcentajes corresponden a los campos agricultura, ganadería, pesca, silvicultura y veterinaria $(0.6 \%)$ y tecnologías de la información y las comunicaciones (TIC), también con $0.6 \%$. Otros campos que cuentan con una mayor presencia de mujeres son salud y servicios sociales (9.9\%), educación (9\%) y artes, humanidades y lenguas (6.4\%) (INE, 2017). Sin embargo, en todas las categorías de profesorado de educación universitaria en las universidades públicas hay mayor participación masculina. Según datos recogidos en el INE (2017), la mayor participación femenina en el profesorado de educación universitaria del curso 2014-2015 corresponde al personal contratado (43.9\%), a los titulares de escuela universitaria $(40.6 \%$ ) y a los titulares de universidad (39.9\%). La participación femenina más baja corresponde a la categoría de catedráticos de universidad $(20.8 \%)$.

Aunque la presencia de la mujer en el mundo científico ha aumentado, muy pocas han gozado de igualdad de oportunidades para aportar su contribución y disfrutar de los beneficios de una carrera científica. Eso es a la vez injusto y poco práctico. Mary Osborn (1992: 101) escribió en una carta publicada en la revista Nature que 
no hay pruebas de que el género esté relacionado con el éxito en la investigación científica y que las mujeres están preparadas para ser juzgadas con los mismos criterios objetivos con los que se valora a sus compañeros varones. Sin embargo, a cambio, las mujeres tienen el derecho de demandar las mismas oportunidades de empleo, los mismos recursos y disfrutar de los mismos privilegios dados a los hombres que se encuentran en una etapa profesional similar.

En el ámbito concreto de la literatura científica, cabe destacar la proliferación de estudios, a nivel internacional, que tienen por objeto analizar el sesgo potencial que el género puede representar en el desarrollo de la carrera científica. La mayoría de los estudios de género en ciencia y tecnología han puesto de relieve el uso insuficiente de recursos humanos, así como la existencia de barreras invisibles al ascenso de las mujeres a las mismas posiciones que sus colegas hombres. Aunque ya no es posible ocultar la creciente participación femenina en este ámbito, aún persisten concepciones y prácticas institucionales que revelan una desvaloración de la mujer (Villarroya Planas et al., 2008). Lo que en el pasado fue simplemente la exclusión explícita de lo femenino en la ciencia hoy se expresa como "techos de cristal" o barreras invisibles que limitan el acceso de las mujeres a los lugares de mayor prestigio y poder de decisión. Este despilfarro de recursos adopta formas diferentes y aparece en distintos momentos de la carrera científica de las mujeres. Para referirse a ellos la literatura científica ha empleado conceptos como "tubería agujereada", "techo de cristal" o "efectos tijeras" (Osca-Lluch, 2011).

Una herramienta que nos permite conocer el estado de las mujeres en la ciencia son los estudios bibliométricos (Torres-Salinas, Muñoz-Muñoz y Jiménez-Contreras, 2011). Los estudios bibliométricos de género posicionan y revelan la situación de las mujeres en la ciencia a través del análisis de sus publicaciones científicas, que son el principal instrumento utilizado por la comunidad investigadora para la difusión del nuevo conocimiento generado en la investigación. Su cometido es visibilizar a las autoras de estos trabajos mediante el estudio de su productividad y el impacto de sus investigaciones y a través del análisis de sus hábitos de investigación en comparación con los de sus homólogos masculinos (Muñoz Muñoz, 2006).

Los trabajos bibliométricos que aplican una perspectiva de género se realizan, en gran parte, con el propósito general de identificar posibles desigualdades entre hombres y mujeres, tanto desde el punto de vista de la autoría de trabajos y producción científica (Barrios, Villarroya y Borrego, 2013; González-Álvarez y Cervera-Crespo, 2017; Mayer et al., 2017; Pyatigorskaya y Di Marco, 2017; Velasco et al., 2014), patrones de colaboración científica (Araujo y Fontainha, 2017; Osca-Lluch, 2012; Peñaranda-Ortega, Osca-Lluch y 
Quiñones-Vidal, 2013), presencia en comités editoriales de revistas científicas (Addis y Villa, 2003; Mauleón et al., 2013; Miqueo et al., 2010, 2011; Ortega et al., 2015; Stegmaier, Palmer y Van Assendelft, 2011), en los órganos de dirección de los colegios profesionales (Botello-Hermosa, Casado-Mejía y Germán-Bes, 2015) o la movilidad de los investigadores (Cañibano, Fox y Otamendi, 2016). Sin embargo, a pesar de que la presencia femenina se ha incrementado en todas las disciplinas, algunos estudios generalistas sobre el estado de la mujer en la ciencia indican que las investigadoras publican menos artículos científicos y firman en posiciones menos relevantes que los investigadores, participan en menos colaboraciones internacionales y reciben un menor número de citas a sus trabajos (FECYT, 2017; Larivière et al., 2013; Skinner y Louw, 2009).

En la actualidad existe un creciente interés en España por mejorar la situación de las mujeres en diferentes ámbitos profesionales, entre los que se incluyen los científicos y académicos (Torres-Salinas, Muñoz-Muñoz y Jiménez-Contreras, 2011). Visibilizar a las mujeres dando a conocer sus aportaciones en el desarrollo de la ciencia y en el avance del conocimiento sigue siendo una actividad imprescindible en el lento camino hacia la igualdad efectiva entre hombres y mujeres. Transcurrida ya la primera década del siglo XXI, la brecha de género continúa estando presente y se manifiesta en múltiples facetas de la vida cotidiana o de las relaciones interpersonales, laborales e institucionales (Barberá Heredia, 2011).

Este estudio pretende analizar, mediante indicadores bibliométricos, la producción científica española en literatura (productividad, colaboración, visibilidad y citación de los trabajos publicados), desagregada según el sexo de sus autores. Los datos obtenidos constituyen una nueva aportación para profundizar sobre la situación de la mujer en la ciencia, proporcionan una información objetiva para conocer los hábitos de publicación de los investigadores en literatura y permiten verificar si existen diferencias significativas en el comportamiento entre hombres y mujeres en esta disciplina.

\section{Metodología}

Para la realización de esta investigación se ha utilizado la base de datos Web of Science (WoS). La metodología utilizada para recoger toda la producción científica en literatura realizada en las instituciones españolas se puede agrupar en cuatro fases: 
En primer lugar, se elaboró una estrategia de búsqueda empleando el término "Literature" en el campo "Topic" y el término "Spain" en el campo "Address". Se revisaron todos los documentos incluidos en la WoS hasta 2017, seleccionando aquellos en los que alguno de los investigadores firmantes del trabajo perteneciera a alguna institución española y que además el trabajo estuviera clasificado en alguna de las áreas temáticas de literatura utilizadas en estas bases de datos. Los datos de toda la producción científica española en literatura se descargaron en una base de datos gestionada en Access, que permitió el tratamiento y análisis de los datos.

En segundo lugar, se efectuó una meticulosa revisión y normalización manual unificando las diferentes variantes de los nombres de un mismo autor o institución, bien porque los autores no firman siempre sus trabajos de la misma forma o por la existencia de algunas erratas en la base de datos en los nombres de los autores e instituciones. En el caso de los autores, el criterio que se siguió ante dos o más variantes de un mismo nombre y/o apellidos consistió en localizar la coincidencia en los lugares de trabajo de las diferentes variantes, o bien consultar el texto completo de los trabajos publicados, donde es habitual que se anote el nombre completo de los autores.

En tercer lugar, se procedió a la asignación del sexo de los autores con base en el nombre completo de los mismos. En algunos casos se tuvieron que consultar las páginas web institucionales o de los autores, dado que el nombre no era suficiente para poder clasificar a los autores por género, o bien se accedió al texto completo de los trabajos o a los índices de las revistas fuente donde fueron publicados. En esta fase se procedió también a la localización y normalización de la institución de trabajo de cada autor.

Por último, se analizaron e identificaron las revistas utilizadas por los investigadores de esta disciplina y se localizaron los datos de las que se encuentran incluidas en los listados de los Journal Citation Reports (JCR).

Los indicadores se calcularon sobre la totalidad de la comunidad investigadora en literatura que desarrolla su trabajo en instituciones españolas, con el objeto de determinar la existencia de diferencias según el sexo. Se analizó la productividad según el número de trabajos, su tipología documental, colaboración científica, revistas de publicación de los trabajos y redes de coautoría de trabajos como base para la identificación de grupos de investigación.

Para gestionar toda la información, normalizar los nombres de los autores y calcular los indicadores bibliométricos y de redes sociales, así como la construcción de las representaciones gráficas de las agrupaciones de autores, se han utilizado los programas Microsoft Office Access para Windows y el programa de análisis y visualización de redes Ucinet. 


\section{Resultados}

Se han recuperado 944 trabajos de literatura publicados en revistas incluidas en la Web of Science (WoS), realizados por 806 autores diferentes que al menos han publicado un trabajo. Se ha identificado el género de la totalidad de los autores. De estos autores, 448 (55.51 \%) son hombres y 358 (44.49\%) son mujeres.

La distribución cronológica (Tabla 1) muestra que hay un aumento en el número de autores que trabajan en instituciones españolas que han realizado algún trabajo de literatura publicado en revistas indexadas en la WoS. Los datos reflejan que hay un incremento constante tanto en el número de hombres como de mujeres, con un predominio significativo de los autores varones hasta el periodo 1998-2002. Se observa que a partir del quinquenio 2003-2007 el número de autoras no ha dejado de crecer, siendo durante el quinquenio 2013-2017 donde hay una menor diferencia entre el número de autores hombres $(51.53 \%)$ y mujeres $(48.47 \%)$.

\begin{tabular}{|c|c|c|c|c|c|}
\hline \multicolumn{7}{|c|}{ Número de autores } \\
\hline Años & Hombres & $\begin{array}{c}\% \\
\text { Hombres }\end{array}$ & Mujeres & $\begin{array}{c}\% \\
\text { Mujeres }\end{array}$ & Total \\
\hline Antes de 1978 & 5 & 83.33 & 1 & 16.67 & 6 \\
\hline $1978-1982$ & 8 & 57.14 & 6 & 42.86 & 14 \\
\hline $1983-1987$ & 5 & 41.67 & 7 & 58.33 & 12 \\
\hline $1988-1992$ & 25 & 80.65 & 6 & 19.35 & 31 \\
\hline $1993-1997$ & 25 & 67.57 & 12 & 32.43 & 37 \\
\hline $1998-2002$ & 78 & 75.73 & 25 & 24.27 & 103 \\
\hline $2003-2007$ & 56 & 56.57 & 43 & 43.43 & 99 \\
\hline $2008-2012$ & 86 & 51.81 & 80 & 48.19 & 166 \\
\hline 2013-2017 & 269 & 51.53 & 253 & 48.47 & 522 \\
\hline Total & 557 & 56.26 & 433 & 43.74 & 990 \\
\hline
\end{tabular}

Tabla 1. Distribución de hombres y mujeres en relación con los años de publicación de los trabajos *El número de autores totales no coincide con el número de autores desglosados por años por el hecho de que hay autores que han publicado en diferentes años, mientras que en total se computan los autores distintos en todo el periodo

En lo que se refiere a la distribución según la tipología documental de los trabajos analizados, puede observarse que los trabajos de literatura indizados en la WoS están clasificados en ocho categorías documentales (Tabla 2). Tal como se observa en la tabla, la tipología documental más frecuente son los artículos y las revisiones de libros. Se observa que la producción científica de 
los hombres supera a la de las mujeres en casi todas las tipologías documentales, excepto en dos categorías: Bibliography y Fiction creative prose.

\begin{tabular}{|l|c|c|c|c|c|c|c|c|c|c|}
\hline \multirow{2}{*}{$\begin{array}{c}\text { Tipología } \\
\text { documental }\end{array}$} & \multicolumn{4}{|c|}{ Número de autores } & \multicolumn{5}{c|}{ Número de trabajos } \\
\cline { 2 - 13 } & $\mathbf{H}$ & $\begin{array}{c}\% \\
\mathbf{H}\end{array}$ & $\mathbf{M}$ & $\begin{array}{c}\% \\
\mathbf{M}\end{array}$ & Total & $\mathbf{H}$ & $\begin{array}{c}\% \\
\mathbf{H}\end{array}$ & $\mathbf{M}$ & $\begin{array}{c}\% \\
\text { M }\end{array}$ & Total \\
\hline Article & 372 & 54.95 & 305 & 45.05 & 677 & 473 & 55.19 & 384 & 44.81 & 857 \\
\hline Bibliography & 4 & 44.44 & 5 & 55.56 & 9 & 4 & 44.44 & 5 & 55.56 & 9 \\
\hline Bibliographical-Item & 2 & 66.67 & 1 & 33.33 & 3 & 2 & 66.67 & 1 & 33.33 & 3 \\
\hline Book Review & 68 & 63.55 & 39 & 36.45 & 107 & 75 & 64.66 & 41 & 35.34 & 116 \\
\hline Editorial Material & 15 & 51.72 & 14 & 48.28 & 29 & 15 & 51.72 & 14 & 48.28 & 29 \\
\hline Fiction, Creative & 0 & 0 & 1 & 100 & 1 & 0 & 0 & 1 & 100 & 1 \\
Prose & 1 & 100 & 0 & 0 & 1 & 1 & 100 & 0 & 0 & 1 \\
\hline Letter & 2 & 66.67 & 1 & 33.33 & 3 & 2 & 66.67 & 1 & 33.33 & 3 \\
\hline Proceedings Paper & 464 & 55.90 & 366 & 44.10 & 830 & 572 & 56.13 & 447 & 43.87 & 1019 \\
\hline Total & & & & & & & & & & \\
\hline
\end{tabular}

Tabla 2. Distribución de hombres y mujeres en relación con la tipología documental de los trabajos *El valor de los autores totales y trabajos no coincide con los valores reales por el hecho de que hay autores que han publicado diferentes tipos de trabajos y hay trabajos que tienen varios autores, y se ha computado el mismo trabajo a cada uno de los autores firmantes. $\mathrm{H}$ : hombres, $\mathrm{M}$ : mujeres

Para determinar las características de la autoría de los trabajos conviene conocer tanto el número total de autores como la cantidad de trabajos que publica cada autor y la distribución del número de autores por trabajo. Hace ya algún tiempo Crump (1984) indicó que el firmante solitario era una especie en extinción. El aumento en el número de autores por trabajo, tanto a nivel nacional como internacional, es un hecho bien constatado (Yegros-Yegros, Tur y Amat, 2012; Wuchty, Jones y Uzzi, 2007). En el caso específico de las disciplinas de las ciencias sociales, la tendencia en el número de autores por trabajo también es creciente, a pesar de presentar un ritmo más lento que otras áreas (Henriksen, 2016) y de que las investigaciones no dependen del equipamiento o del tamaño de los laboratorios.

Cuando se agrupan los autores por sexo y niveles de productividad (Tabla 3), se observa que de los 806 autores que han publicado trabajos de literatura, una gran proporción $(82.75 \%$ ) son transitorios, ya que publican un único trabajo durante todo el periodo. Entre estos autores eventuales o transitorios hay más hombres $(56.31 \%$ ) que mujeres $(43.69 \%$ ). También se observa que, entre los grandes productores (autores con más de cinco trabajos), no hay ninguna mujer y solamente es entre los medianos productores (entre dos y cinco trabajos) donde el número es prácticamente el mismo (69 hombres, $50.36 \%$; 68 mujeres, $49.64 \%$ ). 


\begin{tabular}{cccccc}
$\begin{array}{c}\text { Número de autores } \\
\text { con } \boldsymbol{n} \text { trabajos }\end{array}$ & Hombres & $\%$ & Mujeres & $\%$ & Total \\
\hline 1 trabajo & 375 & 56.31 & 290 & 43.69 & 665 \\
\hline 2 trabajos & 43 & 45.74 & 51 & 54.26 & 94 \\
\hline 3 trabajos & 20 & 62.50 & 12 & 37.50 & 32 \\
\hline 4 trabajos & 5 & 71.43 & 3 & 28.57 & 8 \\
\hline 5 trabajos & 1 & 33.33 & 2 & 66.67 & 3 \\
\hline 6 trabajos & 3 & 100 & 0 & 0 & 3 \\
\hline 8 trabajos & 1 & 100 & 0 & 0 & 1 \\
\hline Total autores & $\mathbf{4 4 8}$ & 55.51 & 358 & $\mathbf{4 4 . 4 9}$ & $\mathbf{8 0 6}$
\end{tabular}

Los autores que destacan por tener una mayor producción son Jon Kortazar (Univ. País Vasco) con ocho trabajos, Fernando Cabo Aseguinolaza (Univ. Santiago de Compostela), Francisco Javier Diez de Revenga (Univ. Murcia) y César Domínguez (Univ. Santiago de Compostela) con seis trabajos cada uno, y María José Olaziregi Alustiza (Univ. A Coruña), Maria do Cebreiro Rabade Villar (Univ. A Coruña) y Javier Sánchez Zapatero (Univ. Salamanca) con cinco trabajos cada uno de ellos.

Un indicador importante relacionado con la autoría y que es utilizado para medir la colaboración científica es la coautoría, es decir, cuando un investigador escribe un documento científico con otro investigador. En las últimas décadas ha aumentado considerablemente la colaboración entre investigadores en muchas áreas de la ciencia, y es un hecho constatado que los artículos individuales están desapareciendo en favor de los grandes grupos en algunas áreas de la ciencia (Yegros-Yegros, Tur y Amat, 2012). Cuando se analiza la colaboración científica en los trabajos de literatura realizados por autores que trabajan en instituciones españolas y que han sido indexados en la WoS, se observa que 882 trabajos $(93.43 \%$ ) han sido realizados individualmente, 55 trabajos $(5.83 \%)$ han sido realizados en colaboración entre dos autores, seis trabajos $(0.64 \%)$ han sido realizados en colaboración entre tres autores y solamente hay un trabajo que ha sido realizado en colaboración entre cuatro autores $(0.11 \%)$.

La Tabla 4 muestra la evolución de los trabajos realizados en colaboración. El análisis de la coautoría revela que los trabajos de literatura realizados por autores que trabajan en instituciones españolas y que se encuentran 
indizados en la WoS han sido realizados en su mayoría con la participación de dos autores. A partir de 2012 se observa que el número de trabajos realizados en colaboración va aumentando, destaca 2016 por ser el año en el que más trabajos se realizaron en colaboración.

\begin{tabular}{|c|c|c|c|c|}
\hline Años & $\begin{array}{c}2 \\
\text { autores }\end{array}$ & $\begin{array}{c}3 \\
\text { autores }\end{array}$ & $\begin{array}{c}4 \\
\text { autores }\end{array}$ & Total trabajos \\
\hline 1991 & 1 & 0 & 0 & 1 \\
\hline 1995 & 1 & 0 & 0 & 1 \\
\hline 1996 & 0 & 0 & 0 & 0 \\
\hline 1997 & 0 & 0 & 0 & 0 \\
\hline 1998 & 0 & 0 & 0 & 0 \\
\hline 1999 & 1 & 0 & 0 & 1 \\
\hline 2000 & 0 & 0 & 0 & 0 \\
\hline 2001 & 1 & 0 & 0 & 1 \\
\hline 2002 & 1 & 0 & 0 & 1 \\
\hline 2003 & 0 & 0 & 0 & 0 \\
\hline 2004 & 1 & 1 & 0 & 2 \\
\hline 2005 & 1 & 0 & 0 & 1 \\
\hline 2006 & 1 & 0 & 0 & 1 \\
\hline 2007 & 2 & 1 & 0 & 3 \\
\hline 2008 & 2 & 0 & 0 & 2 \\
\hline 2009 & 3 & 0 & 0 & 3 \\
\hline 2010 & 0 & 0 & 0 & 0 \\
\hline 2011 & 2 & 0 & 0 & 2 \\
\hline 2012 & 5 & 0 & 0 & 5 \\
\hline 2013 & 6 & 0 & 0 & 6 \\
\hline 2014 & 3 & 1 & 0 & 4 \\
\hline 2015 & 7 & 0 & 1 & 8 \\
\hline 2016 & 12 & 3 & 0 & 15 \\
\hline 2017 & 5 & 0 & 0 & 5 \\
\hline Total & 55 & 6 & 1 & 62 \\
\hline
\end{tabular}

Tabla 4. Evaluación del número de trabajos realizados en colaboración 
La colaboración científica entre los autores y las instituciones es un rasgo diferencial de la investigación que se realiza actualmente. En este sentido, cada vez es más frecuente que los trabajos de investigación sean firmados por un número mayor de autores o instituciones. Esto, que hasta hace relativamente poco tiempo estaba restringido a las ciencias puras, experimentales y a las ingenierías, se está extendiendo, aunque con menor intensidad, a las ciencias sociales y a las humanidades (Durden y Perri, 1995; Endersby, 1996). Una característica definitoria de la colaboración es su relación positiva con la productividad: los investigadores más productivos son los que más colaboran, lo que a su vez tiende a aumentar la visibilidad de esos autores. Esta interesante relación entre la colaboración y la producción científica provoca que una de las principales aplicaciones de la bibliometría sea dicho análisis de colaboración, con el objetivo de detectar los grupos de autores que colaboran entre sí en un periodo temporal o en un área científica dada.

En este trabajo la caracterización global de la colaboración entre los autores se ha efectuado a partir del índice de colaboración o índice firmas por trabajo. Para la identificación de clusters o agrupaciones de autores se han identificado todas las combinaciones de pares de autores presentes en cada uno de los trabajos, es decir, las coautorías. El término coautoría hace referencia a la firma conjunta de dos autores en un mismo trabajo científico. En la Figura 1 se representan de forma diferenciada por sexos las relaciones de coautoría establecidas entre los autores que han realizado algún trabajo de literatura publicado en revistas indexadas en la WoS. Se puede apreciar los principales núcleos de investigadores y las posiciones que ocupan las mujeres en los mismos y los vínculos que han establecido. Se han identificado 16 agrupaciones de autores compuestas por 38 autores. De las 16 agrupaciones se conforman 12 de dos autores, dos de tres autores y dos de cuatro autores. De las agrupaciones con dos autores, hay que destacar que nueve de ellas están formadas por autores del mismo sexo (cinco de sólo hombres y cuatro de sólo mujeres) y tres formadas por un autor de cada sexo (un hombre y un mujer). En el caso de las agrupaciones de mayor tamaño, formadas por tres o cuatro autores, se observa que todas están formadas por equipos mixtos (hombres y mujeres). El número total de equipos mixtos es siete, que reúnen un total de 20 autores (11 hombres y nueve mujeres). Las agrupaciones de cuatro autores tienen como investigadores centrales a Jon Kortazar (Univ.del Pais Vasco) y a Asunción López-Varela Azcarate (Univ. Complutense de Madrid). El grupo liderado por Jon Kortazar (Univ. País Vasco), que es el mayor productor de trabajos de literatura indizados en la WoS durante el periodo estudiado, está formado por profesores de la misma universidad: Ibon Egaña (Univ. País Vasco), Iratxe Retolaza (Univ. País Vasco) y Francisco Javier Rojo Cobos (Univ. País Vasco). 
El otro grupo, liderado por Asunción López-Varela (Univ. Complutense de Madrid), colabora con investigadores de otras instituciones como Maya Zalbidea (Univ. Don Bosco, El Salvador), Ioan-Flaviu Patrunjel (Univ. del Bio-Bio, Chile) y Mark C. Marino (Univ. of Southern California).

En las agrupaciones de tres autores se observa que un grupo está liderado por Moises Selfa (Univ. Lleida), que colabora con Fernando José Fraga de Azevedo (Univ. Minho, Portugal) y Angela Maria Franco Martins Coelho de Paiva Balça (Univ. Evora, Portugal). En el otro grupo formado por tres miembros se observa que la colaboración más intensa se produce entre Amelia Sanz (Univ. Barcelona) y María Goicoechea (Univ. Complutense de Madrid) y que Maria Goicoechea colabora también con Luis Pablo (Univ. Complutense de Madrid). Entre los grupos de dos autores se observa con claridad, dado el grosor de las líneas que unen los nodos que representan a los autores, que la relación más intensa de colaboración se produce entre Javier Calle Martín (Univ. Málaga) y Antonio Miranda García (Univ. Málaga), que han colaborado en la realización de tres trabajos.

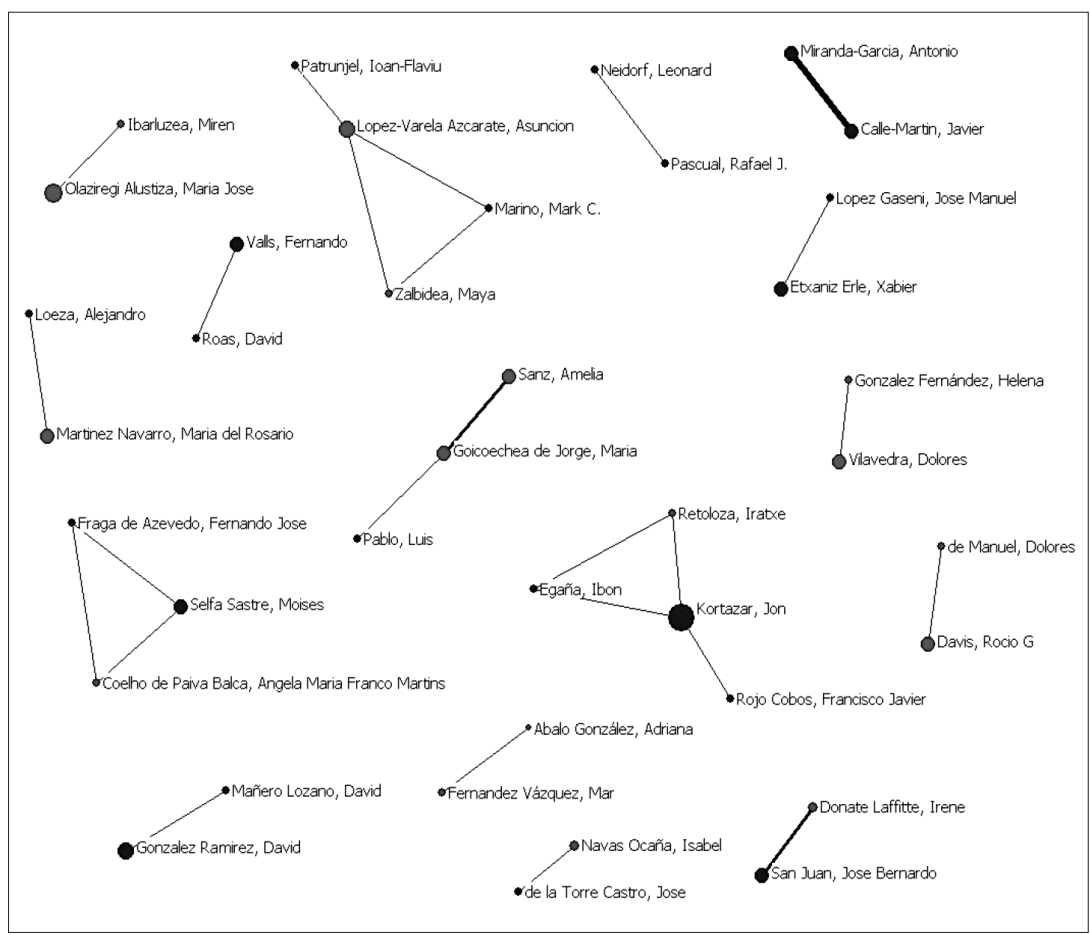




\section{Conclusiones}

Visibilizar a las mujeres dando a conocer sus aportaciones en el desarrollo de la ciencia y el avance del conocimiento sigue siendo una actividad imprescindible. Este estudio pone de manifiesto la utilidad de los indicadores derivados de las publicaciones para conocer la participación de hombres y mujeres en la actividad científica en las diferentes disciplinas e identificar tendencias temporales. Consideramos que su obtención de forma periódica es importante para poder realizar un seguimiento de la participación de las mujeres en las diferentes facetas de la actividad científica. El análisis de la producción científica española en literatura en la base de datos de la Web of Science (WoS) nos permite observar que existe un incremento en el número de trabajos publicados en esta disciplina en revistas que tienen una difusión internacional, se observa que $55.51 \%$ de los autores son hombres y $44.49 \%$ son mujeres. También se observa que, aunque sigue existiendo un alto porcentaje de autores que publican en solitario y que 882 trabajos $(93.43 \%$ ) han sido realizados individualmente, en los últimos años se van incrementado los trabajos realizados en colaboración, tal como sucede en otras disciplinas científicas (González-Alcaide y Gómez Ferri, 2017). Este incremento en la producción científica coincide con el incremento del número de trabajos realizados por mujeres. A partir de 2003 el número de autoras no ha dejado de crecer, siendo durante el quinquenio 2013-2017 donde hay una menor diferencia entre el número de autores $(51.53 \%)$ y autoras $(48.47 \%)$. Cuando se analiza la productividad de los autores que han publicado algún trabajo de literatura durante todo el periodo analizado, se observa que hay una gran proporción $(82.75 \%$ ) de autores eventuales o transitorios, y al clasificar los autores por género vemos que $56.31 \%$ de estos autores son hombres y $43.69 \%$ son mujeres.

Llama la atención que, entre los grandes productores, considerando como tales a los autores que tienen más de cinco trabajos publicados e incluidos en WoS, no hay mujeres y solamente es entre los medianos productores (entre dos y cinco trabajos) donde el número de hombres $(69,50.36 \%$ ) y mujeres $(68,49.64 \%)$ es prácticamente el mismo.

Cuando se analiza la colaboración científica en los trabajos de literatura realizados por autores que trabajan en instituciones españolas y que han sido indexados en la WoS se observa que solamente $5.83 \%$ de los trabajos han sido realizados en colaboración entre dos autores, $0.64 \%$ han sido realizados en colaboración entre tres autores y solamente hay un trabajo que ha sido realizado en colaboración entre cuatro autores $(0.11 \%)$.

A comienzos del siglo XXI, la idea de que mujeres y hombres formen equipos de trabajo diversos, compartan puestos directivos y repartan responsabilidades representa un valor añadido para afrontar la complejidad y 
dinamismo de los entornos globalizados. Sin embargo, hasta el momento no se ha conseguido romper con las asimetrías de género ni con las relaciones de poder entre hombres y mujeres (Barberá Heredia, Ramos López y Candela Agulló, 2011). La incorporación de la mujer a la investigación, la docencia o la gestión de la ciencia supone un progreso social y enriquece el conocimiento. El análisis de redes sociales aplicado a la colaboración en las publicaciones científicas permite identificar los principales grupos y redes de trabajo que están generando la producción científica. En este caso, se han identificado 16 agrupaciones compuestas por un total de 38 autores. Se observa que las agrupaciones que cuentan con un mayor número de autores incluyen todas ellas tanto autores como autoras.

Futuras líneas de trabajo deberían identificar las redes y los grupos que se establecen al analizar las revistas nacionales de esta disciplina y los ámbitos temáticos de investigación de los grupos identificados. Además, dado el carácter dinámico de la ciencia y los grupos de investigación, sería interesante observar su evolución temporal y analizar sus variaciones.

\section{REFERENCIAS}

Addis, E. y P. Villa. 2003. "The editorial boards of italian economics journals: women, gender and social networking”. Feminist Economics 91 (1): 75-91.

Araujo, T. y E. Fontainha. 2017. "The specific shapes of gender imbalance in scientific authorships: A network approach”. Journal of Informetrics, 11: 88-102. http:// dx.doi.org/10.1016/j.jpi.2016.11.002

Barberá Heredia, E. 2011. "Prólogo. La aportación de la mujer a la bistoria de la ciencia y de la técnica en España". Valencia: Instituto de Historia de la Medicina y de la Ciencia López Piñero.

Barberá Heredia, E., A. Ramos López y C. Candela Agulló. 2011. "Laberinto de cristal en el liderazgo de las mujeres”. Psicothema 23 (2): 173-179.

Barrios, M., A. Villarroya y A. Borrego. 2013. "Scientific production in psychology; a gender analysis". Scientometrics, 95: 15-23.

Botello-Hermosa, A., R. Casado Mejía y C. Germán-Bes. 2015. "Presencia de las mujeres en los órganos de dirección de los colegios profesionales del ámbito de la salud en 2015”. Revista Española de Salud Pública, 89, 1-6.

Cañibano, C., M. F. Fox y F. J. Otamendi. 2016. "Gender and patterns of temporary mobility among researchers". Science and Public Policy 43 (3): 320-331.

Crump, A. J. 1984. “They are not alone”. Nature, 309-310.

Durden, G. C. y T. J. Perri. 1995. "Coauthorship and publication efficiency”. Atlantic Economic Journal 23 (1): 69-76.

Endersby, J. W. 1996. "Collaborative research in the Social Sciences: multiple authorship and publication credit”. Social Science Quarterly 77 (2):375-392. 
FECYT (Fundación Española para la Ciencia y la Tecnología). 2017. Indicadores del Sistema español de ciencia, tecnología e innovación. Madrid: Fecyt. http://www.idi. mineco.gob.es/stfls/MICINN/Investigacion/FICHEROS/Indicadores_2017.pdf

González-Alcaide, G. y J. Gómez Ferri. 2017. Análisis de las prácticas de colaboración científica: una vía hacia la excelencia. Valencia: Nau Llibres.

González-Álvarez, J. y T. Cervera-Crespo. 2017. "Research production in high-impact journals of contemporary neuroscience: A gender analysis". Journal of Informetrics, 11, 232-243. http://dx.doi.org/10.1016/j.joi.2016.12.007

González-Sala, F., J. Osca-Lluch, F. Tortosa-Gil y M. Peñaranda-Ortega. 2017. “Characteristics of monographic special issues in Ibero-American psychology journals: visibility and relevance for authors and publishers". Scientometrics 112 (2): 1069-1077. DOI: 10.1007/s11192-017-2372-4

Henriksen, D. 2016. "The rise in co-authorship in the social sciences (1980-2013)". Scientometrics 107 (2): 455-476. https://doi.org/10.1007/s11192-016-1849-x

Huyer, S. y G. Westholm. 2002. Toolkit on Gender Indicators in Engineering, Science and Technology. París: Unesco.

INE (Instituto Nacional de Estadística). 2017. Mujeres y bombres en España 2017. Madrid: Ministerio de Educación, Cultura y Deporte.

Koning, C. J., C. B. Fell, L. Kellnhofer y G. Schui. 2015. "Are there gender differences among researchers from industrial/organizational psychology?". Scientometrics 105 (3): 1931-1952. DOI: 10.1007/s11192-015-1646-y

Larivière, V., Y. Gingras, B. Cronin y C. R. Sugimoto. 2013. "Global gender disparities in science". Nature, 504: 211-213.

Mauleón, E., L. Hillán, L. Moreno, I. Gómez y M. Bordons. 2013. "Assessing gender balance among journal authors and editorial board members". Scientometrics, 95: 87-114.

Mayer, E. N., S. M. Lenherr, H. A. Hanson, T. C. Jessop y W. T. Lowrance. 2017. "Gender differences in publication productivity among academic urologists in the United States". Urology, 103: 39-45. http://dx.doi.org/10.1016/j.urology.2016.12.064

Miqueo, C., C. Germán Bes, T. Fernández Turrado y M. J. Barral Morán. 2011. Ellas también cuentan. Cientificas en los comités de revistas biomédicas. Zaragoza: Prensas Universitarias de Zaragoza PUZ.

Miqueo, C., C. Germán Bes, T. Fernández Turrado y M. J. Barral Morán. 2010. Disparidad de género en los órganos de dirección de revistas biomédicas españolas. Madrid: Instituto de la Mujer. http://www.inmujer.es/documentacion/Documentos/DE0283.pdf

Muñoz Muñoz, A. M. 2006. Presencia y producción cientifica de las profesoras de la Universidad de Granada (1975-1990). Granada: Editorial Universidad de Granada.

Ortega Toro, E., P. Valdivia-Moral, R. González Col y J. L. González Col. 2015. “Género en los comités editoriales y científicos de las revistas españolas de ciencias del deporte". Apunts. Educación Física y Deportes, 120: 67-72.

Osborn, M. 1992. "Letter". Nature 360: 101.

Osca-Lluch, J. 2011. La aportación de la mujer a la historia de la ciencia y de la técnica en España. Valencia: Instituto de Historia de la Ciencia y de la Documentación López Piñero.

Osca-Lluch, J. 2012. "Productividad y colaboración científica desde una perspectiva de género en la Revista Española de Drogodependencias”. Revista Española de Drogodependencias 37 (1): 9-21. 
Peñaranda-Ortega, M., J. Osca-Lluch y E. Quiñones-Vidal. 2013. "La colaboración científica por género en psicología social y de la personalidad: ¿Qué pasa con la colaboración entre grandes productoras?”, en La colaboración cientifica: una aproximación multidisciplinar, Gregorio González, Javier Gómez y Víctor Agulló (coords.), 429-439. Valencia: Nau Llibres.

Pyatigorskaya, N. y L. Di Marco. 2017. "Women authorship in radiology research in France: An analysis of the last three decades". Diagnostic and Interventional Imaging, 98: 769-773.

Skinner, K. y J. Louw. 2009. "The feminization of psychology: data from South Africa”. International Journal of Psychology, 44: 81-92.

Stegmaier, M., B. Palmer y L. Van Assendelft. 2011. "Getting on the board: the presence of women in political science journal editorial positions". PS: Political Science E Politics 44 (4): 799-804.

Torres-Salinas, D., A. M. Muñoz-Muñoz y E. Jiménez-Contreras. 2011. “Análisis bibliométrico de la situación de las mujeres investigadoras de Ciencias Sociales y Jurídicas en España”. Revista Española de Documentación Científica 34 (1): 11-28.

Velasco, J., M. Vilariño, B. G. Amado y F. Fariña. 2014. "Análisis bibliométrico de la investigación española en psicología desde una perspectiva de género”. Revista Iberoamericana de Psicología y Salud 5 (2): 105-118.

Villarroya Planas, A., M. Barrios Cerrejón, A. Borrego Huerta, A. Frías Castillo, C. Olié Castellá y L. Seoané del Moral. 2008. La situación de la mujer en la universidad española: análisis de la producción científica por género (1990-2005). Madrid: Ministerio de Educación y Ciencia.

Wininger, A. E., J. P. Fischer, E. F. Likine, A. S. Gudeman, A. R. Brinker, J. Ryu, K. A. Maupin, S. Lunsford, E. C. Whipple, R. T. Loer, y M. A. Kacena. 2017. "Bibliometric analysis of female authorship trends and collaboration dynamics over JBMR's 30-year history”. Journal of Bone and Mineral Research 32 (12): 2405-2414.

Wutchy, S., B. Jones y B. Uzzi. 2007. "The increasing dominance of teams in production knowledge”. Science, 316, 1036-1039.

Yegros-Yegros, A., E. M. Tur y C. B. Amat. 2012. "Número de autores y colaboración institucional en los artículos originales de investigación biomédica española. Evolución de los valores básicos de referencia en el período 1990-2009”. Medicina Clínica 138 (4): 165-170.

Para citar este texto:

Haba-Osca, Julia, Julia Osca-Lluch y Francisco González-Sala. 2019. "Producción científica española en literatura desde una perspectiva de género a través de Web of Science (1975-2017)". Investigación Bibliotecológica: archivonomía, bibliotecología e información 33 (79): 35-50. http://dx.doi.org/10.22201/iibi.24488321xe.2019.79.57996 